\title{
CREENCIAS SEXISTAS Y PERCEPCIÓN DE ABUSO EN MUJERES ADOLESCENTES Y ADULTAS ${ }^{1}$
}

\author{
Alodia Fernández Puerta ${ }^{2}$ y Marisol Navas Luque ${ }^{3}$
}

\begin{abstract}
Creencias Sexistas y percepción de abuso en mujeres adolescentes y adultas
Resumen: El objetivo del presente estudio es analizar creencias sexistas, creencias sobre la naturaleza del amor y percepción de indicadores de abuso en la pareja, en mujeres de diferentes edades. Participaron 130 mujeres, adolescentes (Grupo $1, \mathrm{n}=65$ ) y mayores de 50 años (Grupo $2, \mathrm{n}=65)$ que cumplimentaron un cuestionario. Los resultados muestran que las adolescentes tienen mayores dificultades al detectar los indicadores de abuso y al percibir abuso encubierto por parte de sus parejas. Sin embargo, no se encontraron diferencias entre ambos grupos en creencias sexistas ni en el apoyo a los mitos sobre el amor romántico.

Palabras clave: creencias sexistas, indicadores de abuso, mitos sobre el amor romántico.
\end{abstract}

\section{Sexist Beliefs and Perception of Violence in Young and Adult Women}

Abstract: This article examines sexist beliefs, assumptions regarding the nature of love and perception of abuse indicators within couples in women of different age groups. In order to do so, 130 women completed a questionnaire. They were divided in two groups: women under 65 years old (Group 1, n =65) and women over 50 years old (Group 2, $n=65$ ). The results show that younger women have more difficulties detecting abuse indicators and perceiving hidden abuse. Nonetheless, no significant differences were found regarding sexist beliefs and romantic love myths in the two age groups.

Keywords: sexist beliefs, abuse indicators, romantic love myths.

\section{Introducción}

Según un informe de la Organización Mundial de la Salud (OMS 2013), "la violencia contra la mujer es un importante problema de salud pública, así como una violación flagrante de los derechos humanos". El informe refleja que al menos un 30\% de las mujeres del mundo han sufrido en alguna ocasión algún tipo de violencia física y/o sexual por parte de sus parejas. Asimismo, constata que el $38 \%$ de los homicidios en mujeres se debe a la violencia conyugal. Actualmente, además, se observa un aumento de violencia en parejas jóvenes, donde los agresores son los chicos y las chicas son las que

${ }^{1}$ Fecha de recepción: 02/07/2016.

Fecha de aceptación: 21/08/2016.

${ }^{2}$ Estudiante del Máster de Psicología Jurídica y Forente, Univesidad de Granada; $₫$ alofp@ @orreo.ugr.es.

${ }^{3}$ Catedrática de Psicología Social, Departamento de Psicología, Universidad de Almería; $\bowtie$ msnavas@ual.es. 
la sufren. La Unidad Didáctica de Prevención de la Violencia de Género en Jóvenes y Adolescentes (2013: 13) afirma que "la violencia en la adolescencia y la juventud es tan severa o más que la que se presenta en la vida adulta y con frecuencia es en el noviazgo cuando va forjándose una relación que se transformara en dramática años más tarde". A raíz de estos datos y de estudios realizados con anterioridad podemos decir que la violencia por razón de género es un problema social que afecta a millones de mujeres en todo el mundo y que va aumentando con los años. Se trata de una violencia que tiene su origen en las desigualdades que existen entre hombres y mujeres y, concretamente, en un sistema de creencias sexistas, que considera y trata a la mujer como inferior al hombre.

El presente trabajo tiene como objetivo fundamental analizar las creencias sexistas en mujeres que han tenido un proceso de socialización diferente por haber nacido en épocas muy distintas: en los años 60 del siglo XX (mujeres adultas mayores de 50 años), y en el año 2000 (mujeres adolescentes). Suponemos que las diferencias en los contextos socio-económicos, educativos, de valores, etc., en los que han crecido estos dos grupos de mujeres, se verán reflejados en diferentes tipos de creencias sexistas, así como en el apoyo a mitos románticos sobre el amor, y en la percepción de indicadores de abuso o maltrato en las relaciones de pareja. Consideramos que el aumento de la violencia de género entre parejas jóvenes o adolescentes puede estar relacionado con las creencias sobre la naturaleza del amor. Es posible que quienes son adolescentes tengan una visión distorsionada del amor, y esto hace que no identifiquen como abuso o maltrato ciertos comportamientos de su pareja que, a la postre, son aceptados y considerados, en muchos casos, una prueba de "amor verdadero". A continuación, se presenta brevemente la justificación teórica del estudio.

1.1. Sexismo y creencias sexistas: la teoría del sexismo ambivalente

El sexismo es uno de los temas más estudiados en Psicología Social en las últimas décadas. Un gran número de autores han abordado este tema desde diferentes perspectivas. En general, existe un acuerdo notable en que el sexismo es una actitud prejuiciosa basada en la creencia de que las mujeres son inferiores como grupo. 
La teoría del sexismo ambivalente (Glick y Fiske 1996) sostiene que existen dos tipos de sexismo. El primero, denominado sexismo hostil, consiste en una actitud negativa basada en la inferioridad de la mujer como grupo y que normalmente va acompañada por estereotipos y discriminación. El segundo, el sexismo benevolente, es aquel que idealiza a las mujeres como amas de casa, madres y objetos románticos, considerándolas inferiores al asumir que necesitan de un hombre para cuidarlas y protegerlas (Cuadrado 2009). Glick y Fiske distinguen tres ideologías de género hostiles y benevolentes dentro de las dos formas de sexismo: el patriarcado o poder estructural masculino, la diferenciación existente entre hombres y mujeres, y la heterosexualidad (Cuadrado 2009).

El patriarcado tiene que ver con el paternalismo y la dominancia del hombre sobre la mujer. Presenta dos componentes o justificaciones: uno hostil y otro benévolo. El paternalismo dominante (hostil) hace referencia a la creencia de que los hombres tienen más poder que las mujeres; por su parte, el paternalismo protector (benévolo) consiste en la percepción de que la mujer debe ser protegida y cuidada por el hombre.

La diferenciación existente entre hombres y mujeres también tiene dos componentes: la diferenciación de género competitiva (hostil) que radica en la inferioridad de la mujer como grupo en cuanto a la competencia, y la diferenciación de género complementaria (benévolo) que reside en los roles establecidos por la sociedad tradicional.

Finalmente, la heterosexualidad también tiene un componente hostil denominado la hostilidad heterosexual, que está basada en la creencia de que las mujeres usan la sexualidad como un medio para manipular a los hombres. El componente benévolo, denominado intimidad heterosexual, hace referencia a la creencia de que la motivación sexual está unida al deseo de los hombres de proximidad psicológica. Esta intimidad está relacionada con las agresiones en las relaciones dentro de la pareja, ya que son un modo de mantener las desigualdades mediante el control. 
1.2. Mitos románticos: creencias sobre la naturaleza del amor

Los mitos sobre el amor romántico son un conjunto de creencias socialmente compartidas sobre la "supuesta verdadera naturaleza del amor" y, al igual que sucede en otros ámbitos, también suelen ser ficticios, absurdos, engañosos, irracionales e imposibles de cumplir (Yela 2003, cit. en Bosch et al. 2007: 28). Estos falsos mitos son producto de la cultura y de ideas preconcebidas acerca de lo que significa enamorarse y amar de forma verdadera. Esta visión errónea conduce a que las jóvenes desarrollen relaciones de pareja disfuncionales y asfixiantes, que favorecen y facilitan la violencia de género y las ideas machistas de la vida cotidiana (Instituto Andaluz de la Mujer 2010). El Cuadro 1 recoge los principales mitos del amor romántico que han sido estudiados (Bosch et al. 2007).

Cuadro 1. Mitos sobre el amor romántico (Bosch et al. 2007)

\begin{tabular}{|ll|}
\hline Mito de la media naranja & $\begin{array}{l}\text { Mitos sobre el amor romántico } \\
\text { pareja predestinada. }\end{array}$ \\
\hline $\begin{array}{l}\text { Mito de la pasión eterna o de } \\
\text { la perdurabilidad }\end{array}$ & $\begin{array}{l}\text { Creencia de que la pasión y el amor de los primeros } \\
\text { meses de relación deben durar toda la vida. }\end{array}$ \\
\hline Mito de la omnipotencia & $\begin{array}{l}\text { Creencia de que "el amor lo puede todo" y, por tanto, } \\
\text { que con amor se podrán superar todos los obstáculos } \\
\text { que se interpongan en la pareja. }\end{array}$ \\
\hline $\begin{array}{l}\text { Mito del matrimonio o de la } \\
\text { convivencia }\end{array}$ & $\begin{array}{l}\text { Creencia de que el amor romántico-pasional debe } \\
\text { llevar a la pareja a formar una unión estable. Este mito } \\
\text { considera que matrimonio y pasión están enlazados, } \\
\text { lo que puede llevar a la decepción. }\end{array}$ \\
\hline $\begin{array}{l}\text { Mito del emparejamiento o de } \\
\text { la pareja }\end{array}$ & $\begin{array}{l}\text { Creencia de que la pareja heterosexual y la } \\
\text { monogamia son algo natural y universal, que se da en } \\
\text { todas las culturas. }\end{array}$ \\
\hline Mito de la exclusividad & $\begin{array}{l}\text { Creencia de que sólo se puede amar a una persona al } \\
\text { mismo tiempo. }\end{array}$ \\
\hline Mito de la fidelidad & Creencia de que el amor supone ser fiel a tu pareja. \\
\hline Mito de los celos & $\begin{array}{l}\text { Creencia de que los celos son una prueba del amor de } \\
\text { la pareja o de que sin ellos no existe amor verdadero. }\end{array}$ \\
\hline Mito de la equivalencia & $\begin{array}{l}\text { Considera que "amor" y "enamoramiento" son } \\
\text { elementos similares, y si uno falla la pareja debe } \\
\text { abandonar la relación. }\end{array}$ \\
\hline
\end{tabular}




\begin{tabular}{|lll|}
\hline $\begin{array}{l}\text { Mito de la ambivalencia } \\
\text { compatibilidad }\end{array}$ & $\begin{array}{l}\text { Consiste en creer que amar a alguien puede ser } \\
\text { compatible con maltratar. Aceptar este mito justifica } \\
\text { la violencia de género. }\end{array}$ \\
\hline Mito del libre albedrío & $\begin{array}{l}\text { Considera que los sentimientos amorosos son íntimos } \\
\text { y no están influidos por factores sociales, biológicos } \\
\text { y culturales ajenos a nuestra voluntad. }\end{array}$ \\
\hline
\end{tabular}

1.3.1. Percepción de abuso y maltrato: indicadores predictivos de la violencia de género

Los estudios han identificado una serie de indicadores que permiten prevenir la violencia en la pareja. En general, hay dos tipos de señales de abuso que aparecen gradualmente en la relación de pareja. Las primeras, denominadas de "abuso encubierto", son sutiles, encubiertas o indirectas, y se dan en las primeras fases de la relación. Se disfrazan con sentimientos amorosos y son muy difíciles de percibir ya que son comportamientos relacionados con el control y posesión que van aumentando con el tiempo. Cuando la víctima no sabe reconocer estas señales de advertencia y sigue con la relación, comienza a justificar al agresor y a culparse por su comportamiento. La conducta se refuerza cuando la víctima acepta las peticiones de cambio y arrepentimiento del maltratador. En esta última fase, los indicadores ya son directos, patentes u hostiles "abuso patente"-(Instituto Andaluz de la Mujer 2010).

Un indicador es una especie de advertencia que, en caso de percibirse, permite a la persona alejarse de la amenaza. En la violencia de género, estos indicadores están relacionados con las estrategias de coacción que emplean los maltratadores para someter a sus parejas. Se trata de los siguientes: Aislar. Consiste en eliminar el apoyo social de la mujer con el fin de hacerla vulnerable. Sin este apoyo de familiares y amigos, la mujer se aísla y se vuelve dependiente del maltratador. Es una de las primeras estrategias de coacción utilizadas por el agresor al comienzo de la relación de maltrato.

Desvalorizar. Consiste en destruir la autoestima y la seguridad en sí misma que posee la víctima, creándole confusión sobre sus propias percepciones. Con esto, la mujer se vuelve más susceptible a ser sometida por su maltratador. 
Amenazar e intimidar. Estas formas de abuso aparecen al final de la fase de conquista o cortejo, y tienen tres objetivos. Por un lado, impedir que la víctima denuncie o cuente los abusos que sufre. Por otro, impedir que deje la relación y crear un sentimiento de culpa en la víctima haciéndole saber que es la razón de su falta de control.

Control, dominio, posesión y celos. Estas conductas tienen lugar al final del ciclo del maltrato. En este punto, tras aislar, destruir la autoestima de la víctima y crear terror, el maltratador ejerce el poder de la relación sin resistencia. La consecuencia para la víctima es falta de libertad física y psíquica, dificultades en la toma dedecisiones y miedo por las consecuencias al no seguir las reglas impuestas por su maltratador.

Coacción o abuso sexual. Los abusos sexuales están recogidos en el Código Penal como delitos contra la libertad o indemnidad sexuales, e incluyen el abuso sexual y la agresión sexual. Según el Instituto de la Mujer y para la Igualdad de Oportunidades (2016) la diferencia entre éstas reside en que "en la agresión sexual se exige que aquél que atenta contra la libertad sexual de la víctima lo haga con violencia o intimidación, mientras que el caso del abuso sexual, la conducta punible se realiza sin violencia e intimidación y sin que medie consentimiento".

Violencia física. Incluye una amplia gama de agresiones (bofetadas, quemaduras, empujones, zarandeos, patadas, etc.) hasta el homicidio, y los objetivos son similares a los anteriores, es decir, el control por parte del maltratador.

Chantaje emocional. Este tipo de abuso se da en la fase de arrepentimiento de la relación. Consiste en manipular y doblegar la voluntad de la víctima mediante el uso de técnicas de coacción. El objetivo final es parecer arrepentido y vulnerable a ojos de la víctima para que ésta no abandone la relación.

Manifestación de creencias sexistas. Por último, este factor de riesgo se basa en manifestaciones de ideas y creencias de que las mujeres son inferiores a los hombres, y como tales, deben obedecer. Son explícitas y pueden manifestarse en insultos injustificados hacia las mujeres. 
La violencia en la pareja no nace de forma espontánea, sino que existen una serie de indicadores de abuso que nos permiten reconocer a un maltratador. El problema es que no muchas mujeres saben detectarlo a tiempo ya que muchos de estos comportamientos son tan sutiles que la propia víctima no puede distinguirlos.

\subsection{El presente estudio}

El presente trabajo pretende comparar las creencias sexistas, el apoyo a mitos románticos y la capacidad para detectar indicadores de abuso o maltrato por parte la pareja, en dos grupos diferentes de mujeres a las que se le suponen experiencias educativas, socializadoras y de vida muy diferentes en función de su edad: mujeres adultas mayores de 50 años, y mujeres adolescentes.

Por un lado, suponemos que las mujeres adultas (mayores de 50 años) puntuarán más alto en sexismo benevolente que las adolescentes (Hipótesis 1). Esta hipótesis se apoya en la creencia de que las mujeres que nacieron en una sociedad más tradicional pueden tener una visión más estereotipada y sexista que las adolescentes sobre cómo son y cómo deberían ser hombres y mujeres, los roles que deberían desempeñar, o cómo deben ser las relaciones entre ambos sexos.

Por otro lado, suponemos que las adolescentes tendrán una visión inmadura del amor y, por tanto, aceptarán como verdaderos una serie de mitos románticos, mientras que las mujeres adultas, precisamente por su edad y por sus diferentes experiencias, no presentarían ningún tipo de apoyo a estos mitos (Hipótesis 2). Es decir, se espera que las adolescentes puntúen más alto en mitos románticos que las mujeres adultas.

Por último, suponemos que las adolescentes tendrán más problemas para percibir el abuso que las mujeres adultas (Hipótesis 3) debido a las creencias, basadas en mitos románticos, que tienen sobre el amor. Por ello, restarían importancia a los comportamientos de abuso y los achacarían a comportamientos propios del "amor verdadero". 
En resumen, en este trabajo pretendemos conocer si existen diferencias en cuanto a creencias sexistas, creencias sobre la naturaleza del amor, y detección de indicadores de abuso o maltrato en la relación de pareja, entre dos grupos de mujeres de edades, experiencias y socialización muy distintas. Suponemos que las mujeres adultas tendrán creencias más sexistas que las adolescentes, pero su percepción sobre el amor romántico será más madura que el de las jóvenes y tendrán mayor capacidad para percibir el abuso o maltrato.

\section{Método}

\subsection{Participantes}

La muestra está formada por 130 mujeres, con edades comprendidas entre los 16 y 18 años $(n=65$, Grupo 1; edad media $16.78, D T=.74)$; y entre los 50 y 60 años de edad ( $n=65$, Grupo 2; edad media 54.83, DT=4.05). El grupo de mujeres adolescentes (Grupo1) procede de un Instituto de Enseñanza Secundaria de la provincia de Granada. En el momento en el que se aplicó el cuestionario estudiaban primero o segundo curso de Bachillerato. Por su parte, las mujeres de entre 50 y 60 años (Grupo 2), proceden de diferentes ciudades del país. En lo que se refiere al nivel de estudios, esta muestra está formada por mujeres con estudios universitarios (el 56.9\%), formación profesional (13.9\%), estudios secundarios (15.3\%) y estudios primarios (13.9\%). En cuanto a su estado civil, las mujeres estaban mayoritariamente casadas (75.3\%), el 18.5\% solteras y el $6.2 \%$ con pareja de hecho.

\subsection{Instrumentos}

Las variables del estudio y los instrumentos para medirlas son los siguientes:

Creencias sexistas. Se midió con la Escala de Detección de Sexismo en Adolescentes (DSA; Recio, Cuadrado y Ramos 2007), formada por 26 ítems (10 miden sexismo benévolo y 16 sexismo hostil). La escala de respuesta es tipo Likert con 6 puntos, 
oscilando desde 1(totalmente en desacuerdo) hasta 6 (totalmente de acuerdo). Altas puntuaciones en esta escala y subescalas son indicativas de un mayor sexismo (hostil o benevolente).

Mitos sobre el amor romántico. Se midió con la Escala de Mitos Románticos (Ferrer, Bosch y Navarro 2010), compuesta por 10 ítems que evalúan siete mitos románticos presentados a través de descripciones o frases hechas. La escala de respuesta es tipo Likert con cinco opciones de respuesta, desde 1 (completamente en desacuerdo), hasta 5 (completamente de acuerdo). Altas puntuaciones en esta escala indican mayor apoyo a los mitos del amor romántico.

Percepción de abuso. Se mide con la Escala de Percepción de Abuso (Ramos, Luzón, Recio y de la Peña 2010). El objetivo de este instrumento es conocer si quienes participan realizan una valoración adecuada sobre la presencia o no de abuso o maltrato en las relaciones de pareja y si son capaces de detectar aquellos enunciados que expresan de manera sutil o encubierta el abuso. La escala está formada por 38 ítems, donde se le pide a la persona participante que indique, utilizando una escala de 5 puntos, su valoración de cada uno de los enunciados, oscilando desde 1 (nada de abuso o maltrato) a 5 (totalmente abuso o maltrato). Además, se incluyen elementos neutros para obtener un mayor control interno, que ayuda a dar validez a las respuestas. Cada enunciado hace referencia a diferentes estrategias de coacción utilizadas paras ejercer el abuso o maltrato. Altas puntuaciones en esta escala y en cada subescala indican una mayor percepción de abuso o maltrato.

Variables sociodemográficas. Se midieron las variables sociodemográficas habituales: edad, sexo, nivel de estudios, estado civil y localidad de residencia.

\subsection{Procedimiento}

La selección del grupo de mujeres adolescentes se realizó en un centro de Enseñanza Secundaria. Una vez conseguidos los permisos pertinentes, el cuestionario se aplicó, por parte de la primera autora del trabajo, en cinco clases de Bachillerato, con una 
duración aproximada de 20 minutos por clase. El grupo de mujeres adultas se seleccionó a través de un muestreo de conveniencia, aplicando el cuestionario de manera individual a mujeres del entorno de las autoras del trabajo y utilizando la técnica de la bola de nieve. En este caso, el cuestionario tuvo una duración aproximada de 15 minutos. En ambos grupos las participantes fueron informadas del carácter anónimo de sus respuestas, se les pidió la máxima sinceridad, y fueron informadas brevemente de los objetivos del estudio al finalizar. Asimismo, se agradeció su participación.

\subsection{Análisis de datos}

En primer lugar, se calculó la consistencia interna de cada escala en cada grupo utilizando el coeficiente de estimación de la fiabilidad alpha de Cronbach. En segundo lugar, se analizaron los supuestos paramétricos correspondientes. En tercer lugar, se obtuvieron los estadísticos descriptivos habituales en cada una de las variables y para cada uno de los grupos. Por último, se compararon las puntuaciones medias de ambos grupos en cada escala, utilizando la prueba $t$ de Student. Todos los análisis se realizaron con el programa SPSS 22.

\section{Resultados}

\section{Estimación de consistencia interna}

La escala DSA y la Escala de Percepción de Abuso muestran coeficientes de estimación de la fiabilidad bastante aceptables en el total de cada escala en ambos grupos de mujeres (entre 87 y .96). La Escala de Mitos sobre el amor romántico muestra un alpha de Cronbach más bajo que las otras dos (entre .64 y .69) (véase Tabla 1).

Las correlaciones ítem-total son altas en general, salvo por un ítem de la Escala de Mitos románticos (ítem 30) que mostró una correlación negativa (-.32) y que afectaba 
claramente a la estimación de fiabilidad de la escala. Se decidió eliminarlo y, como resultado, el alpha de Cronbach de esta escala aumentó considerablemente (de .55 a .67).

Tabla 1. Estimación de fiabilidad (alpha de Cronbach) de las escalas DSA, Mitos sobre el amor romántico y Percepción de abuso

\begin{tabular}{lcccccc}
\hline & \multicolumn{2}{c}{$\begin{array}{c}\text { Mujeres } \\
\text { adolescentes }\end{array}$} & \multicolumn{2}{c}{ Mujeres adultas } & \multicolumn{2}{c}{ Total } \\
\hline & $N$ ítems & $\alpha$ & $N$ ítems & $\alpha$ & $N$ ítems & $\alpha$ \\
\hline DSA & 26 & .87 & 26 & .94 & 26 & .92 \\
$\begin{array}{l}\text { Mitos } \\
\text { románticos }\end{array}$ & 10 & .64 & 10 & .69 & 10 & .67 \\
$\begin{array}{l}\text { Percepción } \\
\text { de abuso }\end{array}$ & 38 & .95 & 38 & .95 & 38 & .96 \\
\hline
\end{tabular}

\section{Estadísticos descriptivos}

En las Tablas 2 y 3 se presentan los estadísticos descriptivos obtenidos por ambos grupos de participantes (mujeres adultas y adolescentes) en las tres variables del estudio.

En relación a las creencias sexistas, los resultados muestran que, en contra de lo esperado (Hipótesis 1), no hay diferencias estadísticamente significativas entre ambos grupos de mujeres ni en el total de la escala, $t(128)=1.31, p=.19$, ni en ninguno de sus componentes: sexismo benévolo, $t(128)=1.01, p=.32$, o sexismo hostil, $t(128)=1.37$, $p=.17$. En cuanto a los mitos sobre el amor romántico, en contra también de lo esperado (Hipótesis 2), tampoco se hallaron diferencias estadísticamente significativas entre ambos grupos de mujeres, $t(128)=-1.81, p=.07$, si bien las diferencias son marginalmente significativas, puntuando más alto las chicas adolescentes. 
Tabla 2. Estadísticos descriptivos de mujeres (adultas y adolescentes) en creencias sexistas (sexismo hostil, benevolente y escala DSA) y mitos sobre el amor romántico

\begin{tabular}{lcccc}
\hline & \multicolumn{2}{c}{ Mujeres adultas } & \multicolumn{2}{c}{ Mujeres adolescentes } \\
\hline & $M$ & $D T$ & $M$ & $D T$ \\
\hline Sex.Benevolente & 2.61 & .90 & 2.80 & 1.21 \\
Sex. Hostil & 1.35 & .42 & 1.50 & .78 \\
Total DSA & 1.83 & .54 & 2.00 & .87 \\
Mitos & 2.47 & .53 & 2.28 & .65 \\
románticos & & & & \\
\hline
\end{tabular}

Nota. Creencias Sexistas: las puntuaciones oscilan de 1 a 6 . A mayor puntuación, mayor sexismo.

Nota. Mitos románticos: las puntuaciones oscilan de 1 a 5. A mayor puntuación, mayor aceptación de mitos románticos.

Tabla 3. Estadísticos descriptivos de mujeres (adultas y adolescentes) en percepción de abuso y maltrato

\begin{tabular}{lcccc}
\hline & \multicolumn{2}{c}{ Mujeres adolescentes } & \multicolumn{2}{c}{ Mujeres adultas } \\
\hline & $M$ & $D T$ & $M$ & $D T$ \\
\hline Abuso patente & 4.37 & .70 & 4.55 & .66 \\
Abuso encubierto & 3.32 & .76 & 4.00 & .78 \\
Abuso sexual & 4.27 & .76 & 4.52 & .81 \\
Desvalorizar & 4.05 & .85 & 4.46 & .76 \\
Dominar & 3.61 & .78 & 4.17 & .72 \\
Controlar & 3.81 & .85 & 4.23. & .84 \\
Aislamiento & 3.35 & .76 & 3.80 & .92 \\
Posesión & 3.79 & .85 & 4.33 & .81 \\
Chantaje emocional & 3.14 & .84 & 3.96 & .97 \\
Intimidación & 4.70 & .68 & 4.72 & .66 \\
Agresiones físicas & 4.65 & .72 & 4.78 & .76 \\
Manifestar & 4.30 & .83 & 4.65 & .64 \\
creencias sexistas & & & & \\
Total abuso & 3.85 & .69 & 4.28 & .69
\end{tabular}

Nota. Las puntuaciones oscilan de 1 a 5. A mayor puntuación, mayor capacidad de percepción de abuso o maltrato.

En relación a la percepción de abuso y maltrato (Hipótesis 3), existen diferencias estadísticamente significativas entre ambos grupos de mujeres tanto en el total de la escala, $t(128)=3.53, p<.05$, como en la subescala de abuso encubierto, $t(128)=5.04, p$ 
$<.05$, siendo las mujeres adultas las que perciben mayor abuso total y encubierto. No se encontraron diferencias entre ambos grupos en la subescala de abuso patente, $t(128)=1.5$, $p=.14$.

En cuanto a los indicadores de abuso, se encontraron diferencias estadísticamente significativas en desvalorizar, $t(128)=2.86, p<.05$, dominar, $t(128)=4.22, p<.05$, controlar, $t(128)=2.83, p<.05$, aislamiento, $t(128)=3.09, p<.05$, posesión, $t(128)=$ $3.69, p<.05$, chantaje emocional, $t(128)=5.12, p<.05$, y manifestar creencias sexistas, $t(128)=2.65, p<.05$, obteniendo las mujeres adultas mayor puntuación que las adolescentes en todos ellos, es decir, en la capacidad para considerar ciertos comportamientos como ese tipo de abuso o maltrato. La excepción la encontramos en abuso sexual, $t(128)=1.79, p=.07$, intimidaciones, $t(128)=.13, p=.90$, y agresiones físicas, $t(128)=1.07, p=.29$, donde las puntuaciones de ambos grupos de mujeres son semejantes. En la Figura 1 se muestran gráficamente los resultados obtenidos en cada uno de los indicadores de abuso, en función del grupo.

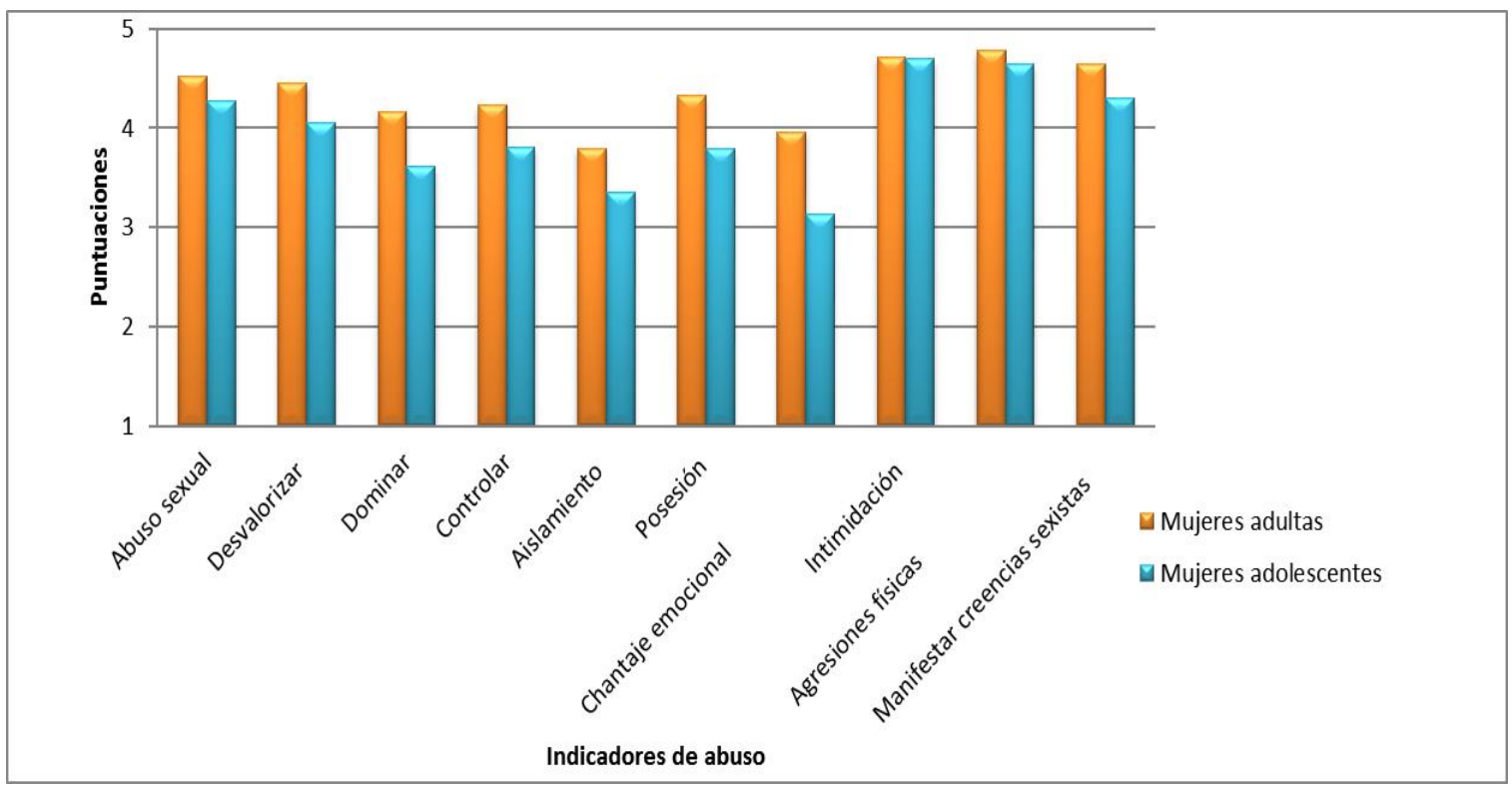

Figura 1. Puntuaciones obtenidas por mujeres adultas y adolescentes en los indicadores de abuso y maltrato. 


\section{Discusión}

El objetivo general de este trabajo era analizar las creencias sexistas en mujeres nacidas en épocas distintas y con un proceso de socialización diferente. Además, analizamos otras variables, como los mitos sobre el amor romántico y la percepción de abuso o maltrato, que podían ser factores a tener en cuenta en el aumento de la violencia en parejas jóvenes en los últimos años. En primer lugar, creíamos que las mujeres adultas obtendrían mayores puntuaciones en creencias sexistas. En segundo lugar, pensábamos que las adolescentes apoyarían en mayor medida los diferentes mitos sobre la naturaleza del amor. Por último, suponíamos que las adolescentes tendrían más dificultades al percibir los indicadores de abuso o maltrato.

Por una parte, los resultados indican que las escalas utilizadas para este estudio tienen una adecuada consistencia interna, aunque la escala de mitos románticos, tiene un índice más bajo, pero aceptable. Este resultado es similar al que obtuvieron en el estudio original Ferrer, Bosch y Navarro (2010), a pesar de tener un tamaño muestral considerable.

En contra de la primera hipótesis, los resultados indican que no hay diferencias estadísticamente significativas entre las mujeres adultas y adolescentes en sus creencias sexistas (ni hostiles, ni benevolentes). Así, las participantes de este estudio apoyan casi con la misma intensidad las creencias sexistas, a pesar de la diferencia de edad y de las diferentes experiencias educativas y socializadoras que se le suponen. En contra de nuestra segunda hipótesis, tampoco se encontraron diferencias estadísticamente significativas en los mitos sobre el amor romántico entre las mujeres adultas y adolescentes. Esperábamos que las adolescentes aceptaran como "verdaderos" los mitos románticos en mayor medida que las mujeres adultas. Sin embargo, a pesar de la superioridad de puntuaciones por parte de las adolescentes, no se puede afirmar que las diferencias no sean debidas al azar, debido a que la probabilidad es sólo marginalmente significativa.

Por último, en cuanto a la tercera hipótesis, los resultados obtenidos en percepción de abuso y maltrato sí muestran diferencias estadísticamente significativas en el total de 
la escala, el abuso encubierto y en la mayoría de los indicadores de abuso analizados. Este estudio revela que las adolescentes tienen mayores dificultades para percibir el abuso encubierto que las mujeres adultas. Asimismo, tampoco perciben otros indicadores de abuso. Sólo la percepción de abuso sexual, intimidación y agresión física son similares en ambos grupos de mujeres. Esto puede deberse a que son comportamientos directos y muestran la violencia de forma tan obvia que detectarlos es más sencillo.

Las diferencias entre ambos grupos subrayan una menor capacidad de las jóvenes ante las señales de advertencia de abusos en las relaciones de pareja. Por tanto, se deberían hacer intervenciones que educaran a los adolescentes a distinguir el abuso. Ayudar a las jóvenes a detectar rápidamente un acto inofensivo, de un indicador de abuso que puede desembocar en violencia de género.

En referencia a las limitaciones de este trabajo, cabe decir que la muestra es pequeña y no representativa de la población, por lo que impide la generalización de resultados. Otra limitación es que ambos grupos de participantes no se seleccionaron de la misma forma por lo que sus resultados no son estrictamente comparables. Finalmente, el nivel de estudios del grupo de mujeres adultas podría influir en las creencias sexistas y en la percepción de abuso. No obstante, consideramos que este trabajo, a pesar de sus limitaciones, aporta aspectos interesantes al estudio del sexismo y la predicción de la violencia de género.

\section{Conclusión}

En definitiva, en las variables de creencias sexistas y mitos sobre el amor romántico no se aprecian diferencias entre los grupos de mujeres. Por lo tanto, no podemos determinar que el sistema de creencias de ambos grupos sea diferente debido a la edad o a las experiencias educativas o de socialización vividas. En cuanto a la percepción de abuso y maltrato, sí encontramos diferencias entre ambos grupos. Las mujeres mayores detectan mejor el abuso. Sin embargo, las adolescentes, aunque no tienen grandes dificultades para percibir el abuso patente, sí las tienen para detectar el 
abuso encubierto en comparación con las mujeres adultas. Esto mismo sucede con la mayoría de los indicadores de abuso. Por tanto, si se desea eliminar la violencia de género, que cada vez es mayor en parejas jóvenes, se deberían realizar intervenciones que enseñaran a las más jóvenes a identificar correctamente los comportamientos y actitudes de abuso de sus parejas.

\section{Referencias bibliográficas}

Bosch, Esperanza, Victoria Ferrer, Esther García, $\mathrm{M}^{\mathrm{a}}$ Carmen Ramis, $\mathrm{M}^{\mathrm{a}}$ Carmen Mas, , Capilla Navarro y Gema Torrens. 2007. Del mito del amor romántico a la violencia contra las mujeres en la pareja. Madrid: Instituto de La Mujer. Ministerio de Igualdad.

Cuadrado, Isabel. 2009. "El estudio psicosocial del prejuicio". Introducción a la psicología social. Coords. Elena Gaviria, Isabel Cuadrado y Mercedes López. Madrid: Sanz y Torres. 411-416.

Expósito, Francisca, Miguel Moya y Peter Glick. 1998. "Sexismo ambivalente: medición y correlatos". Revista de Psicología Social, vol. 2: 159-169.

Ferrer, Victoria, Esperanza Bosch y Capilla Navarro. 2010. "Los mitos románticos en España”. Boletín de Psicología 99: 7-31.

Glick, Peter y Fiske, Susan. (1996). "The ambivalent sexism inventory: Differentiating hostile and benevolent sexism". Journal of Personality and Social Psychology, vol. 70: 491-512.

Instituto Andaluz de la Mujer. 2010. Proyecto Andalucía Detecta. Prevención de la Violencia de Género. Consejería para la Igualdad y Bienestar Social. Junta de Andalucía.

http://www.fundacionmujeres.es/documents/view/informe_andalucia_detecta_and alucia_previene.html [2 marzo 2016]

Instituto de la Mujer y para la Igualdad de Oportunidades. 2016. ¿Qué diferencia la agresión sexual del abuso sexual? http://www.inmujer.gob.es/conoceDerechos/preguntas/delitos.htm $\quad\left[\begin{array}{ll}12 & \text { mayo }\end{array}\right.$ 2016.]

Organización Mundial de la Salud. 2013. Estimaciones mundiales y regionales de la violencia contra la mujer: prevalencia y efectos de la violencia conyugal y de la violencia sexual no conyugal en la salud. http://apps.who.int/iris/bitstream/10665/85243/1/WHO_RHR_HRP_13.06_spa.pd f [12 mayo 2016] 
Ramos, Esther, José María Luzón, Patricia Recio y Eva María de la Peña. 2010. Escala de Percepción de Abuso. Proyecto Andalucía Detecta. Prevención de la Violencia de Género. Sevilla: Instituto de la Mujer. Consejería para la Igualdad y Bienestar Social. Junta de Andalucía.

Recio, Patricia, Cuadrado, Isabel y Ramos, Esther. (2007). "Propiedades psicométricas de la Escala de Detección de Sexismo en Adolescentes (DSA)". Psicothema, vol. 19: 522-528.

Unidad Didáctica de Prevención de la Violencia de Género en Jóvenes y Adolescentes. (2013). Prevención de la Violencia de Género en Jóvenes y Adolescentes. Dirección General de Prevención de Violencia de Género, Juventud, Protección Jurídica y Reforma de Menores. Comunidad Autónoma de la Región de Murcia. http://www.educarenigualdad.org/media/pdf/uploaded/material/306_u-dviolencia-de-gxnero-secundaria-pdf.pdf [30 mayo 2016]. 